\title{
Interaction between Arsenic and Aluminum in Germanium
}

\author{
J. O. McCALDIN \\ Hughes Semiconductors, Newport Beach, California
}

(Received May 28, 1959)

\begin{abstract}
The behavior of $\mathrm{As}$ in $\mathrm{Ge}$ containing regions doped with $\sim 5 \times 10^{20} / \mathrm{cc} \mathrm{Al}$ was studied. The solubility of As is enhanced tenfold or more by the heavy Al doping, on the basis of (1) measurements of conductivity type and (2) the negative results of a search for compounds by $x$-ray diffraction. The behavior of As diffusion fronts was studied by observing the progress of the $p-n$ junction formed in Ge containing $10^{17} / \mathrm{cc}$ In. When a region of heavy $\mathrm{Al}$ doping was added, the $p-n$ junction was displaced. The displacements indicate that the diffusing As is attracted to regions of heavy Al doping. These results are similar to those of Reiss, Fuller, and others for $\mathrm{Li}$ in $\mathrm{Si}$, though a detailed understanding is not yet available in the present case.
\end{abstract}

\section{INTRODUCTION}

$T$ HE simplest treatment of impurity diffusion in a solid assumes that the only driving force for diffusion is the gradient in the impurity concentration. This assumption, which is formulated in Fick's law, is adequate for most cases of diffusion doping of silicon and germanium with impurities from columns III and V. At sufficiently high doping concentrations, however, other driving forces may be expected to become important. For example, in the so-called "double $D$ " effect, for which some evidence has been presented, ${ }^{1}$ the charge interaction in extrinsic regions of a semiconductor provides an additional driving force. Furthermore, an effect corresponding to more than a doubling in the diffusivity might be expected at the junction between two extrinsic regions of opposite type.

The behavior of a semiconducting medium in which two impurities interact has been studied in detail for the case of lithium in doped silicon. These studies, which are most recently described in articles by Fuller and Reiss, ${ }^{2}$ provide a relatively complete and quantitative picture of the system. Perhaps analogous behavior occurs for elements from columns III and V under suitable conditions. The purpose of the present study is to explore this possibility.

\section{SYSTEM Ge-Al-As}

The system Ge-Al-As, which was selected for experiment, offers some distinct advantages for diffusion measurements. The principal advantage is that the diffusivity of As is 100 times or more the diffusivity of $\mathrm{Al}$ in Ge. Thus, to a first approximation, the $\mathrm{Al}$ stands still while the As diffuses. This approximation is basic to the experimental arrangements to be described. A second advantage derives from the high solubilities ${ }^{3,4}$ of both diffusants. Interaction of diffusants is more

' A. D. Kurtz and C. L. Gravel, J. Appl. Phys. 29, 1456 (1958). Also F. M. Smits, Proc. Inst. Radio Engrs. 46, 1049 (1958).

${ }^{2}$ C. S. Fuller and H. Reiss in Semiconductors, edited by N. B. Hannay (Reinhold Publishing Corporation, New York, 1959), Chaps. 5 and 6.

3 'Thurmond, Trumbore, and Kowalchik, J. Chem. Phys. 25, 799 (1956).

4. A. Trumbore and A. A. Tartaglia, J. Appl. Phys. 29, 1511 (1958). likely at high concentrations, and this is particularly important in view of the high temperature needed for measurable diffusion in this system.

The impurities are easily introduced into Ge. Aluminum was alloyed ${ }^{5}$ in by the well-known evaporativefusion process. The Ge was held at about $600^{\circ} \mathrm{C}$ during fusion, so that approximately $5 \times 10^{20} / \mathrm{cc} \mathrm{Al}$ was present in all the regrowth layers to be described. Arsenic was introduced from the vapor established by a doping source consisting of $10^{19} / \mathrm{cc}$ As in Ge. The Ge used in the experiments was indium doped with $C_{b}=10^{17} / \mathrm{cc}$.

\section{SOLUBILITY EFFECTS}

Indications of an interaction between $\mathrm{As}$ and $\mathrm{Al}$ impurities in Ge were first obtained in solubility measurements. A mutual solubility effect of the two impurities is closely related to a diffusion effect. Suppose, for example, that the solubility of As is greater in heavily doped regions of $\mathrm{Ge}$ than in intrinsic regions. An As concentration gradient is present in an equilibrium situation. Fick's law is inadequate in this case, and the heavy doping must be taken into account to describe the diffusion of As.

The starting material in the solubility study was a slice of Ge about $\frac{1}{2} \times 1 \times 0.030$ in. Aluminum was evaporated onto the heated slice to form an $\mathrm{Al}-\mathrm{Ge}$ eutectic structure and an $\mathrm{Al}$ regrow th layer. The eutectic structure had to be completely removed to avoid later complications, and this requirement was met by alternately boiling the slice in $\mathrm{HCl}$ and rubbing the surface to break off the dendrites of the eutectic structure. The regrowth layer which remained was about $15 \times 10^{-4} \mathrm{~cm}$ thick. The slice was cut into dice $0.3 \times 0.3 \times 0.07 \mathrm{~cm}$. Each die was placed with two doping dice, containing $10^{19} / \mathrm{cc} \mathrm{As}$, in an evacuated quart $z$ capsule. The capsule was held at $800^{\circ} \mathrm{C}$ for a selected time and then cooled rapidly. Type measurements were made on the regrowth surface with a thermoelectric probe.

Typically the regrowth surface would remain $p$ type for about $15 \mathrm{hr}$ of the treatment. At this time $n$-type areas developed at various spots on the surface. After

${ }^{5}$ R. A. Gudmundsen and J. Maserjian, Jr., J. Appl. Phys. 28, 1308 (1957). 
some 30 to $60 \mathrm{hr}$ the whole regrowth surface would be $n$ type. The conversion to $n$ type was limited to a thin surface layer at this stage, however, since the lightest polishing of this surface would expose $p$-type material. Conversion of the entire regrowth layer to $n$ type occurred after 200 to $400 \mathrm{hr}$ at $800^{\circ} \mathrm{C}$. At this time an As diffusion front appeared in the bulk Ge just inside the regrowth layer.

This result could not have been anticipated from the solubilities of the individual impurities in Ge. The concentration of As in the doping source used in the present experiments was only $\sim 6 \%$ the concentration of $\mathrm{Al}$ in the regrowth layer. Evidently the As solubility is enhanced some tenfold or more by the presence of the high Al concentration. The over-doping effect is reversible, as can be observed by substituting a getter for the As doping source. Under these conditions the regrowth surface returns to $p$ type.

$\mathrm{X}$-ray diffraction methods were used to examine the completely converted regrowth layer by Dr. John Bryden of this laboratory, who could find no indication of a second phase. Thus it seems likely that aggregation of the As and $\mathrm{Al}$ impurities, if it occurred, was limited to small clusters, perhaps pairs.

\section{DIFFUSION OF AI AND As AT A CORNER}

The behavior of the two impurities during diffusion was first studied with the procedures indicated in Fig. 1. The specimens were Ge dice $0.3 \times 0.3 \times 0.07 \mathrm{~cm}$. An Al
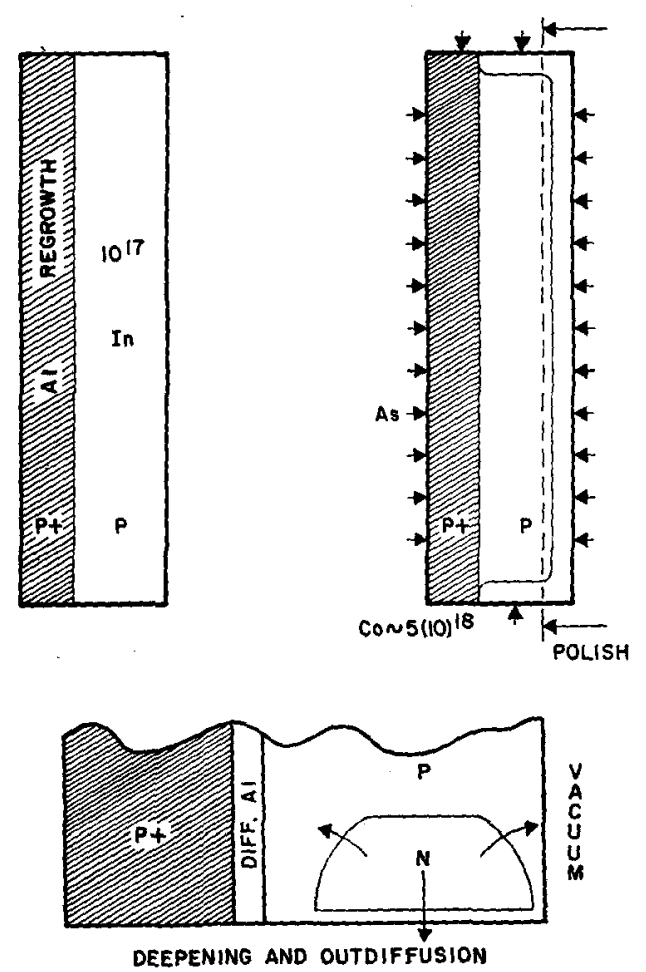

FIG. 1. Preparation of specimens for the corner diffusion experiment. The specimens are shown in cross section. regrowth layer had been provided on one face of each die, as described in Sec. II, except that the regrowth layer in this case was $100 \times 10^{-4} \mathrm{~cm}$ or more in thickness. The regrowth layer, which is labeled $P^{+}$in the figure, was doped about 5000 times more heavily than the bulk $\mathrm{Ge}$, which is labeled $P$. Five dice were sealed in an evacuated quartz capsule together with an equal number of doping dice (Ge with $10^{19} / \mathrm{cc} \mathrm{As}$ ). The capsule was held at $850^{\circ} \mathrm{C}$ for the initial doping period, $\tau \cong 20$ hr. The As atmosphere provided by the doping dice produced a diffused As layer in the specimen dice. On the $P^{+}$side of a specimen die the diffused As caused a spotty surface conversion of type, as indicated in Sec. II. Resistivity profiles made on the $P$ side showed that As was distributed according to a complementary error function with $5 \times 10^{18} \leq C_{0} \leq 10^{19} / \mathrm{cc}$. The face of the (lightly doped) $P$ side of the die was polished to remove the As diffusion front there. Arsenic remained around the edge of the die as shown in Fig. 1.

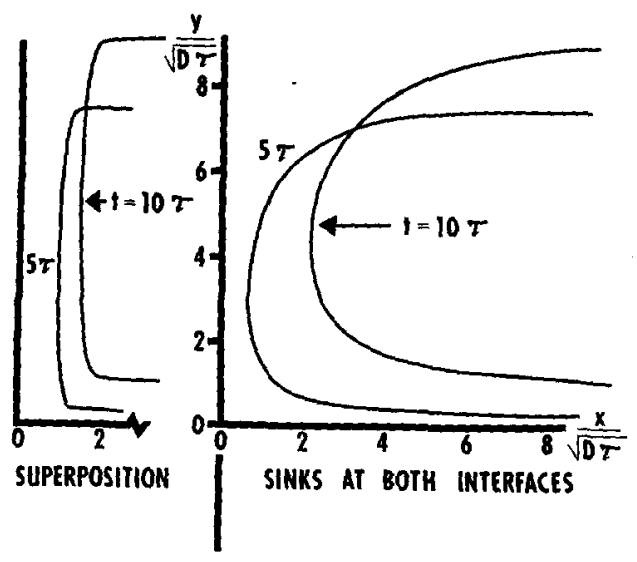

FIG. 2. Junction shapes calculated for corner diffusion. For the right-hand curves, a perfect sink for As is assumed along both coordinate axes. For the left-hand curves, a sink is assumed only along $y=0$, but the spreading out of an Al diffusion front from $x=0$ is allowed for.

The point of interest is the behavior of the diffused As near the $P-P+$ interface. The situation at the edge of a die is shown in the last part of Fig. 1. Neglecting for the moment the diffusion of $\mathrm{Al}$ from the $P+$ region, the $p-n$ junction between the diffused arsenic and the remainder of the die is a contour of equal As concentration, $N_{D}=10^{17} / \mathrm{cc}$. Assuming that Fick's law applies to regions of this order of concentration, the flow of As will be everywhere perpendicular to the junction line. Thus, if the junction curves near the $P-P+$ interface as shown in the figure, As flows preferentially toward the $P+$ regions as indicated. Similarly the vacuum interface on the right operates as a sink for diffusing As. Thus the relative attractions of a vacuum and of a $P+$ region for diffusing As can be compared in the experiment.

The junction curvature that is being sought in the experiment is enhanced if the initial doping period, $\tau$, 
is followed by a deepening period during which sinks for As are effective. During the deepening period the die should be surrounded by vacuum so that outdiffusion occurs at all surfaces of the specimen. Thus the lower right-hand corner of the die shown in Fig. 1 would have sinks for As along both vertical and horizontal boundaries. A similar corner would occur at the left side of the As-rich region if the $P-P+$ interface were as effective a sink for As as the vacuum interface.

The junction shape expected when a perfect sink for As operates along both surfaces at a corner may be calculated. The concentration, $C$, of As at any time, $t$, and place is given by

$$
\frac{C}{C_{0}}=\left(\operatorname{erfc} \frac{y}{2(D t)^{\frac{3}{2}}}-\operatorname{erfc} \frac{y}{2 D^{\frac{1}{2}(t-\tau)^{\frac{1}{1}}}}\right) \operatorname{erf} \frac{x}{2(D t)^{\frac{1}{2}}},
$$

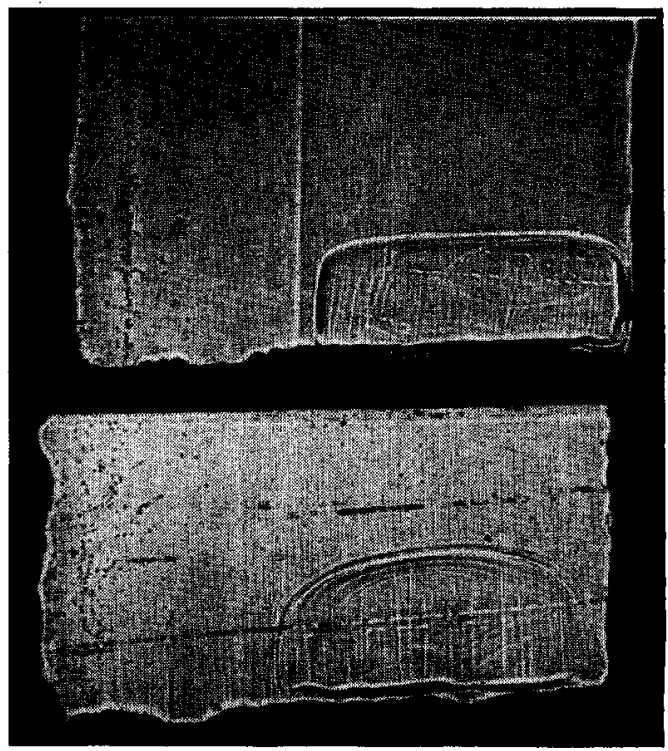

Fig. 3. Actual junction shapes developed during corner diffusion. The upper photomicrograph is for a total time at $850^{\circ} \mathrm{C}$ of $t=4 \tau$. The lower photomicrograph is for $t=11 \tau$. Total width of specimens is about $0.07 \mathrm{~cm}$.

where $D$ is the As diffusivity and $\mathrm{C}_{0}$ is the surface concentration at the end of the initial doping time, $\tau$. To obtain the junction contour the concentration $C$ is set equal to the bulk doping, $N_{A}=10^{17} / \mathrm{cc}$, and a family of curves plotted for various times, $t$. Some typical results of the calculations are shown on the right-hand side of Fig. 2. After deepening times of the order of $10 \tau$, the junction becomes markedly curved.

For comparison, the junction shape expected under the usual assumptions, which neglect an attractive force between $\mathrm{As}$ and $\mathrm{Al}$, is shown on the left-hand side of Fig. 2. In this case the spreading out of $\mathrm{Al}$ from the $P+$ region due to diffusion is taken into account, and the only sink for As is the vacuum interface at $y=0$. Since $D_{\mathrm{As}} \geq 100 D_{\mathrm{Al}}$, the arsenic concentration falls off roughly 10 times more slowly than the $\mathrm{Al}$ concentration, so that
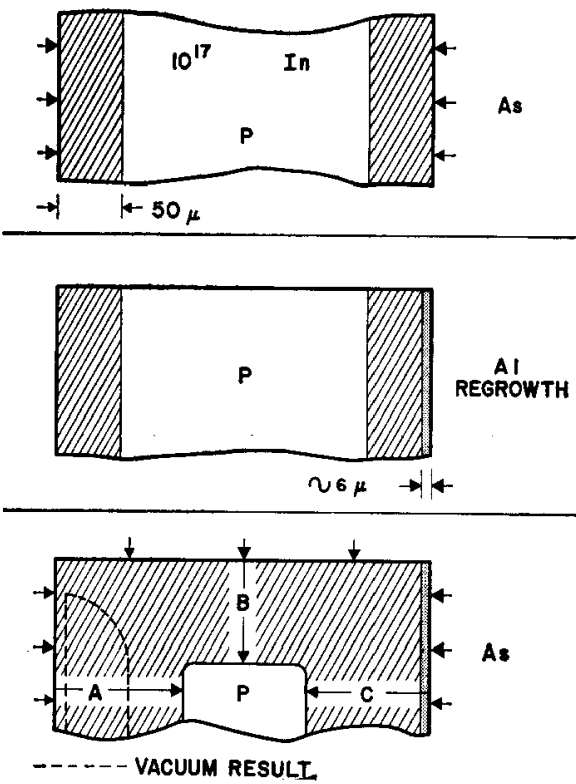

FIG. 4. Preparation of specimens for parallel diffusion experiment. The specimens are shown in cross section.

either one or the other diffusant dominates the junction shape in most regions. The result is an almost rectangular junction shape.

The purpose of the experiment was to decide which of the two calculated junction shapes* actually would develop. Typical metallographic sections obtained under the conditions described are shown in Fig. 3. Early in the deepening period the actual junction shape is hard to classify between the two calculated shapes, though close inspection suggests the interaction assumption is the better of the two. Also note the symmetry in the junction shape between the $P-P+$ interface side and the vacuum interface side. At later stages in the deepening, the junction clearly develops the shape predicted by the interaction hypothesis.

\section{DIFFUSION WITH Al AND As FRONTS PARALLEL}

Other observations of the two impurities during diffusion were made in an essentially one-dimensional situation. The experimental procedure is illustrated in in Fig. 4. Again the starting material was a Ge slice doped with $10^{17} / \mathrm{cc}$ of In. In this case, the As doping was performed first, followed by the formation of an Al regrowth layer. The techniques for carrying out the two processes were very similar to those described in Sec. III, except that a diffusion temperature of $800^{\circ} \mathrm{C}$ and an initial doping time, $\tau$, of about $50 \mathrm{hr}$ were used. The regrowth layer was $10 \%$ to $15 \%$ the thickness of the As layer in which it was formed. After formation of the

* The actual outdiffusion probably is not to a perfect sink as assumed in the calculations. See, for example, R. C. Miller and F. M. Smits [Phys. Rev. 107, 65 (1957)], who studied surface rate limitations on out-diffusion. The junction shape might then be expected to develop more slowly than calculated. 


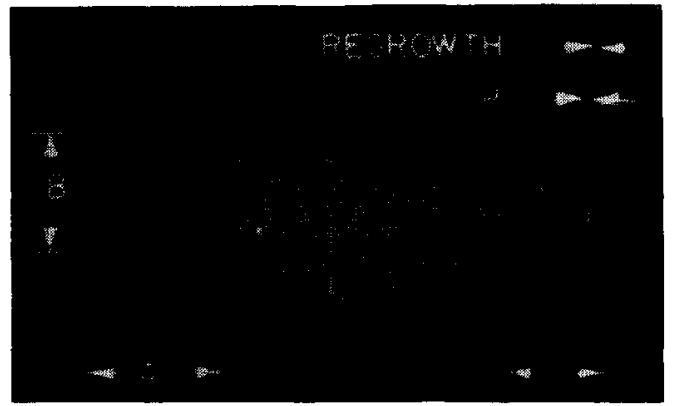

FIG. 5. Metallographic section of a specimen in the parallel diffusion experiment. The dimensions $A, B$, and $C$ are penetrations of As diffusion fronts for various boundary conditions.

regrowth layer, the slice was cut into dice, $0.3 \mathrm{~cm} \times 0.3$ $\mathrm{cm}$. The appearance of the edge of a die was as shown in part 2 of Fig. 4.

The final step in the experiment was a deepening of the As diffusion fronts. Most of the deepening was performed with As vapor over the specimen. Three specimen dice and one doping die were sealed in an evacuated quartz tube, which was heated at $800^{\circ} \mathrm{C}$ for the deepening time. The additional doping source was approximately sufficient to make up for As losses in the system, so that the amount of As in each specimen die, exclusive of the regrowth region, remained roughly constant during deepening. Sheet conductance measurements ${ }^{6}$ made on the die surface with a four-point probe confirmed that the amount of As in the region of interest was constant within $\pm 30 \%$ in every case and within $\pm 15 \%$ in most cases.

The penetration of As that occurred under these conditions is shown schematically in the last part of Fig. 4. The dimension $A$ is the depth of As diffusion through the left-hand surface in the figure. A Gaussian distribution would be expected for this As front. The dimension $B$ is the depth of penetration of As through the freshly cut edge of the die, and for long deepening times is very nearly the same as $A$. The dimension $C$ is the penetration that occurs when a regrowth layer overlying an As diffusion front imposes a boundary condition on it. If $C$ were to lag behind $A$ and $B$, the imposed boundary condition would evidently result in a loss of As from the diffusion front to the regrowth layer.

Figure 5 is a metallographic section showing the experimental result obtained. In this example, the deepening time was about $15 \tau$, i.e., some $780 \mathrm{hr}$, and the deepening temperature was the same as in the initial doping, i.e., $800^{\circ} \mathrm{C}$. The dimensions $A$ and $B$ are nearly equal, as would be expected if these regions were in good contact through a vapor phase. The dimension $C$, however, is only some $70 \%$ as large as $A$ or $B$. Evidently the diffusion front associated with $C$ was weakened by loss of As.

An interesting detail in Fig. 5 is the curvature of the junction at the interior corners. The junction near the

\footnotetext{
B G. Backenstoss, Bell System Tech. J. 37, 699 (1958).
}

weak As front on the right exhibits the greater rounding of the two. A simple argument provides a comparison of the strengths of the As fronts on the basis of the rounding of the junction line. Suppose that one tries to superpose the junction shapes at the two interior corners by a suitable mirror reflection. The discrepancy between the two curves can be ascribed mostly to different As gradients in the horizontal direction. The reasons for this are (1) that superposition of a vertical and a horizontal component of As concentration is a reasonable approximation near the junction, and (2) that the vertical component of As concentration is roughly the same at both interior corners. The validity of the superposition assumption can be judged for the left corner, where the equation of the junction line is

$$
\frac{C}{C_{0}}=\frac{10^{17}}{10^{19}}=\operatorname{erfc} \frac{x}{2(D t)^{\frac{1}{2}}}+\operatorname{erfc} \frac{y}{2(D t)^{\frac{1}{2}}}-\operatorname{erfc} \frac{x}{2(D t)^{\frac{1}{2}}} \operatorname{erfc} \frac{y}{2(D t)^{\frac{1}{2}}}
$$

and the higher order term is less than one percent of the linear terms. The second assumption can be judged in terms of the results obtained in Sec. III. On these assumptions, the gradient in As concentration at the junction is two or three times greater for front $A$ than for front $C$.

In some cases the deepening step in this experiment was performed with the specimen in vacuum. The specimen was sealed in an evacuated quart $z$ capsule together with a getter consisting of pure Al supported on a body of pure Ge. The capsule was heated at $800^{\circ} \mathrm{C}$ for the deepening time. Some of the As penetration distances shown in Fig. 4 were strongly affected by the substitution of vacuum for As vapor. The diffusion front associated with dimension $B$ never formed, and the front $A$ was weakened by loss of As at the surface. Front $C$ was imperceptibly affected.

The progress of the diffusion fronts for the various boundary conditions is shown in Fig. 6. For comparison, the penetration distances for a complementary error function distribution and a Gaussian distribution are also shown. $\dagger$ The erfc distribution corresponds to a small influx of $A$ s at the surface, while the Gaussian distribution corresponds to zero As transfer at the surface. Figure 6 shows that the normal diffusion fronts $A$ and $B$ behave appropriately for a Gaussian or erfc distribution, whereas the diffusion fronts subjected to the boundary condition of a vacuum or an $\mathrm{Al}$ regrowth become weakened.

\section{DISCUSSION}

The solubility and diffusion experiments showed an attractive force to exist between two common dopants of opposite conductivity type, under suitable conditions.

$\dagger$ A curve for the case of outdiffusion to a perfect sink can be made to fit the experimental points for the vacuum boundary contion, provided suitable values of $C_{0}$ and $C_{b}$ are chosen. In the absence of an accurate value for $C_{0}$, however, the curve is not shown. 


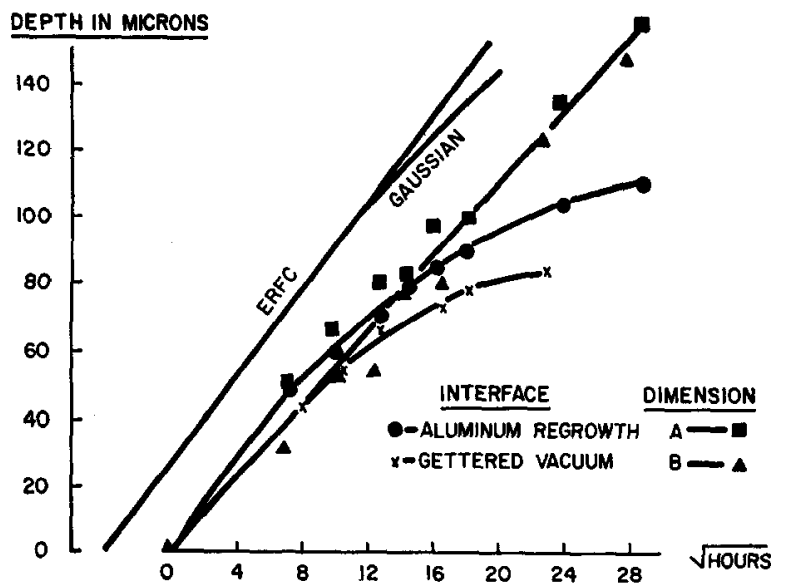

FIG. 6. Penetration of As diffusion fronts for various boundary conditions.

A possible explanation for the attraction is the existence of a macroscopic compound, i.e., second phase, in the ternary system at the compositions and pressures used. The search for such a compound by x-ray diffraction techniques yielded negative results. Furthermore, the diagrams of related binary systems ${ }^{7}$ for which detailed information is available suggest that most III-V compounds probably are not stable at the compositions and relatively low pressures used in the present study. In the absence of an equilibrium diagram for this ternary system, however, the existance of the compound AlAs in the range of composition, temperature, and pressure studied is not altogether ruled out.

Another possibility is the formation of substitutional AlAs pairs, as suggested by Reiss ${ }^{8}$ et al. Such pairs might aggregate to form a macroscopic second phase only after prolonged heating at high temperature, if at all. In support of this possibility, the solubility measurements showed As and Al to be present in a ratio only slightly greater than $1: 1$. Also, diffusing As was observed to permeate a thin $\mathrm{Al}$-rich region relatively slowly. This last observation can be interpreted in terms of a reduced diffusivity, $D^{\prime}$, due to a pairing reaction. For the present experimental values, the reduced value, $D^{\prime}$, is about 0.1 of the normal diffusivity, $D$, for unpaired As. According to the simple theory ${ }^{2}$ for diffusion in the presence of pairing, this result means that most $(\sim 90 \%)$ As atoms are paired, and only the relatively few unpaired atoms are free to diffuse.

A similar experiment has been reported by Fuller. ${ }^{2}$ Radioactive As was the diffusant and heavy doping was obtained with Ga concentrations up to $10^{19} / \mathrm{cc}$, instead of $\mathrm{Al}$ doping. No change in As diffusivity due to $\mathrm{Ga}$ doping was detected. This negative result probably is

${ }^{7}$ J. van den Boomgaard and K. Schol, Philips Research Repts. 12, 127 (1957).

${ }^{8}$ Reiss, Fuller, and Morin, Bell System Tech. J. 35, 535 (1956), Sec. XV. due to the use of an acceptor doping some 50 times less than in the present work. If the same equilibrium pairing constant, $\Omega p$, is applied to the $\mathrm{Al}$ and $\mathrm{Ga}$ cases, the simple theory predicts that $D^{\prime}$ would be about $0.8 \times D$, when $N_{A}$ is $10^{19} / \mathrm{cc}$. This small a change in diffusivity would be difficult to detect.

An alternative interpretation of the present results is that the region of heavy $\mathrm{Al}$ doping remains extrinsic at higher temperatures. Evidence for extrinsic behavior of heavily doped Ge at elevated temperatures has been given by Valenta and Ramasastry ${ }^{9}$ in their study of $\mathrm{Ge}$ self-diffusion. Data ${ }^{10}$ on the hole concentration, $p_{i}$, in intrinsic $\mathrm{Ge}$ suggest an extrapolated value at $850^{\circ} \mathrm{C}$ of $p_{i} \sim 10^{19} / \mathrm{cc}$. The $\mathrm{Al}$ concentration is some 50 times larger, and room temperature measurements ${ }^{4}$ have shown that each acceptor atom gives rise to one hole. Thus the hole concentration is estimated to be $\sim 50$ times larger in the Al-rich region than in the intrinsic region. In terms of the mass action law for the holeelectron equilibrium, the equilibrium concentration of the ionized donor, $\mathrm{As}^{+}$, would also be $\sim 50$ times larger in the Al-rich region. (Actually the mass action law is only an approximation ${ }^{2}$ at these high concentrations.) This distribution of As between the two regions is in qualitative agreeement with the experimental results for solubility and diffusion. The enhanced solubility observed is the right order of magnitude, and the attraction between $\mathrm{As}$ and $\mathrm{Al}$ during diffusion follows directly from the enhanced solubility. The relatively long time required for As to "penetrate" a thin Al-rich region would be due, in this interpretation, to the distribution coefficient of $\sim 50: 1$ in effect at the junction between extrinsic and intrinsic regions.

One way to distinguish between the two interpretations presented is to measure As diffusivity within the Al-rich region, rather than an over-all time for As to penetrate the whole region. The techniques of metallography and resistivity profiling used in the present investigation are not well suited to this purpose. Some profiling measurements were made and inconclusive results obtained, though some slight preference might be given to the substitutional pairing hypotheses on this basis. Other experimental techniques appear more promising, such as the use of tracers and the application of the electron probe microanalyzer ${ }^{11}$ to impurity profiling. The latter technique is being used in this laboratory for further study of the problem.

\section{CONCLUSIONS}

The experiments indicate that arsenic diffusing in germanium at $\sim 800^{\circ} \mathrm{C}$ is attracted to regions of heavy aluminum doping. These regions also exhibit an en-

M. W. Valenta and C. Ramasastry, Phys. Rev. 106, 73 (1957).

${ }^{10}$ F. J. Morin and J. P. Maita, Phys. Rev. 94, 1525 (1954).

11 D. B. Wittry, J. Appl. Phys. 29, 1543 (1958). 
hanced solubility for arsenic. No evidence for the presence of a macroscopic second phase was found; however, other explanations of the observed effects are possible, including: (1) formation of substitutional AlAs pairs; and (2) the persistence of extrinsic behavior to high temperature.

\section{ACKNOWLEDGMENTS}

The author wishes to thank T. W. Griswold and $\mathrm{H}$. Winston for helpful suggestions and R. D. Mathis, J. Healy, H. Ross, and J. Blackmon for assistance with the experiments. Thanks are due to J. Bryden, who made the $x$-ray diffraction examinations.

\title{
Dislocations in Two Types of CdS Crystals*
}

\author{
D. C. Reynolds, Aeronautical Research Laboratory, Wright Patterson Air Force Base, Ohio \\ AND
}

\section{S. J. Czyzaא, Physics Department, University of Detroit, Detroit 21, Michigan}

(Received July 21, 1959)

\begin{abstract}
Dislocation densities in CdS crystals (types I and II) have been investigated employing chemical etching techniques. There is very little difference in the dislocation densities in the two types of CdS crystals. The dislocation density in both types of growth varies over a range from $10^{7} / \mathrm{cm}^{2}$ to $10^{1} / \mathrm{cm}^{2}$. However, type I crystals have a greater tendency to twin and low angle boundaries are generally present in the twinned crystal.
\end{abstract}

\section{INTRODUCTION}

$T$ WO types of crystals are formed from the vapor phase growth of cadmium sulfide. ${ }^{1}$

(a) Type I crystals are those which grow directly on the powder charge with the growth plane parallel to the growth surface.

(b) Type II crystals are those which grow on a seed plate with the grow th plane making an angle of approximately $14^{\circ}$ with the growth surface.

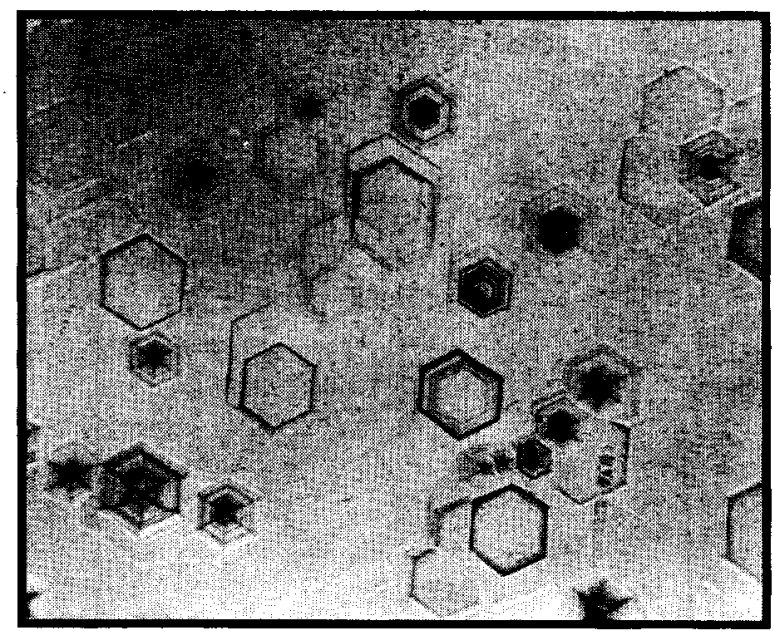

FiG. 1. Typical etch pattern showing the direction of prefer. ential etching ( $c$ axis). Magnification 132X.

* The research effort at the University of Detroit, Physics Department is supported by the Physics Branch ONR Contract No. Nonr 1511 (01) NR 015-218.

${ }_{1}^{1}$ Greene, Reynolds, Czyzak, and Baker, J. Chem. Phys. 29, 1375 (1958).
In both types of growth, the growth plane is the 0001 plane. Dislocation studies have been made on both types of growth using chemical etching techniques.

\section{HYDROCHLORIC ACID ETCHING}

The etching is accomplished by exposing the crystal to hydrochloric acid vapor. In these experiments boiling concentrated hydrochloric acid was used and the crystal was held in the vapor 1 to $2 \mathrm{in}$. above the boiling acid for a period of 5 to $10 \mathrm{sec}$. The etch products are easily removed from the crystal, hydrogen sulfide going off as a gas and cadmium chloride being water soluble. The etch is preferential in the direction of the " $a$ " axis and

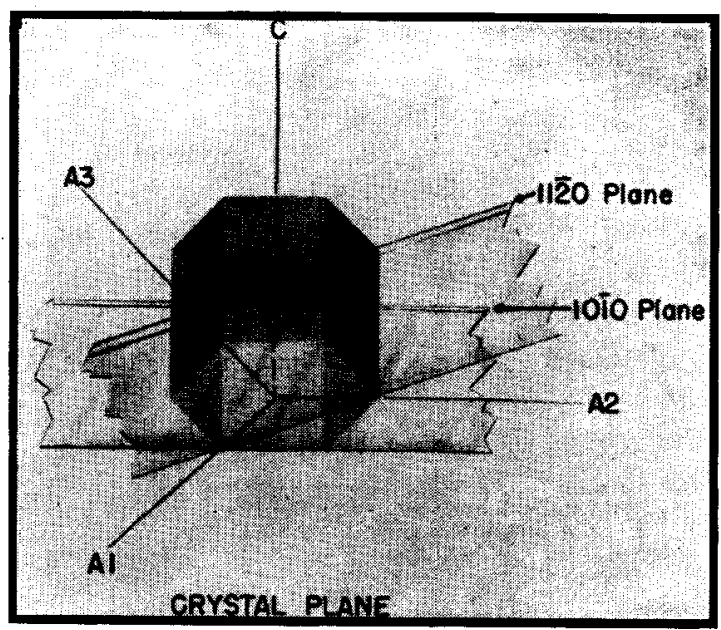

FIc. 2. A pictorial view of $\mathrm{CdS}$ structure. (Consider the light colored spheres to be sulfur and the darker colored spheres to be cadmium.) Magnification $132 \times$. 